\title{
Long-term prognosis and management of hepatocellular carcinoma after curative treatment
}

\author{
Naoshi Nishida \\ Department of Gastroenterology and Hepatology, Kindai University Faculty of Medicine, Osaka, Japan
}

Keywords: Carcinoma, Hepatocellular; Recurrence; Epidemiology; Chemotherapy, Adjuvant; Immunotherapy

\section{See Article on Page 516}

Hepatocellular carcinoma (HCC) is one of the most common cancer and leading cause of related mortalities worldwide. Receiving curative treatment such as radiofrequency ablation (RFA), hepatic resection, and liver transplantation is crucial for long-term survival of HCC patients; however, compared to other cancer types, recurrence after curative treatment is quite common in HCC cases, ${ }^{1}$ thereby having an adverse impact on survival. Therefore, identifying a subset of HCC that carries a high risk of recurrence and development of adjuvant therapy for such cases is clinically important for improving prognosis in the HCC patients.

Notably, HCC recurrence is of two types, metastatic recurrence of the primary lesion and de novo emergence of HCC that attributes to the multicentric occurrence of tumors occurring due to prolonged liver damage such as chronic infection of hepatitis $B$ virus (HBV) and hepatitis C virus (HCV). ' Compared to other cancer types, late recurrence after curative treatment, which is more common for multicentric occurrence, is one of the characteristics of HCC, which should be attributed to the field of carcinogenesis, where the genetic and epigenetic changes occur in hepatocytes of damaged liver. Reportedly, 5- and 10-year distant recurrence rates were $74.8 \%$ and $80.8 \%$, respectively, in the Japanese HCC cohort who underwent RFA. ${ }^{2}$ Moreover, even after controlling hepatitis virus by eliminating HCV and suppressing HBV replication via antiviral therapy, the liver is prone to damage that mainly attributes to the metabolic and life style factors, including alcohol intake, steatosis of the liver due to diabetes mellitus, and obesity. ${ }^{3}$ Thus, long-term monitoring of HCC recurrence is essential even after curative treatment.

Kim et al. ${ }^{4}$ examined the recurrence rate of $\mathrm{HCC}$ for more than 5 years after RFA or resection for early-stage HCC by Barcelona Clinic Liver Cancer Staging system using the large data set from the HCC registry in Korea. They presented a high recurrence rate; the cumulative recurrence rates increased to $39.7 \%, 60.3 \%$, and $71.0 \%$ at 2,5 , and 10 years, respectively, and did not reach a plateau, suggesting that patients were required to continue HCC surveillance even after 5 recurrence-free years. Moreover, they reported that male sex, higher fibrosis-4 (FIB-4) scores, and a-fetoprotein (AFP) levels at 5 years were associated with late $\mathrm{HCC}$ recurrence among patients who did not experience recur-

\section{Abbreviations: \\ AFP, a-fetoprotein; FIB-4, fibrosis-4; HBV, hepatitis B virus; HCC, Hepatocellular carcinoma; $\mathrm{HCV}$, hepatitis $C$ virus; RFA, radiofrequency ablation}

\section{Corresponding author : Naoshi Nishida}

Department of Gastroenterology and Hepatology, Kindai University Faculty of Medicine, 377-2 Ohnohigashi, Osaka-Sayama City, Osaka 5898511, Japan

Tel: +81-72-366-0221 (Ext. 3525), Fax: +81-72-367-2880

E-mail:naoshi@med.kindai.ac.jp

https://orcid.org/0000-0002-9111-5668 
rence for more than 5 years. ${ }^{4}$

For effective monitoring of HCC recurrence, it is crucial to identify the high-risk groups. In general, it is considered that early recurrence is a consequence of primary tumor metastasis, where the aggressive tumor characteristics and treatment modality are critical. Indeed, Kim et al. ${ }^{4}$ presented certain factors related to the risk of tumor metastasis, such as tumor burden and initial treatment modality, as independent factors for recurrence within 5 years after curative treatment. Moreover, our group reported that acculturation of genetic and epigenetic alterations in primary tumor, which are generally related to tumor aggressiveness, efficiently predicts the recurrence after liver transplantation, ${ }^{5}$ thereby suggesting that the primary tumor characteristics are essential for emergence of metastatic recurrence. In contrast, late recurrence is known to reflect the background condition of carcinogenic potential of the liver, where chronic inflammation and tissue damage lead to genetic and epigenetic alterations and HCC recurrence. ${ }^{6}$ For example, oxidative DNA damage caused by chronic inflammation could induce such alterations in the hepatocyte and trigger malignant transformation. ${ }^{7.8}$ Kim et al. ${ }^{4}$ also identified the subgroup that carries high risk of recurrence after 5 recurrence-free years using the factors that reflect the condition of background liver, which include male sex, high FIB-4 score, and AFP levels above $10 \mathrm{ng} / \mathrm{mL}$; accumulation of these risk factors is related to the late-recurrence of HCC. Therefore, type and duration of recurrence should be considered for the surveillance strategy.

To predict the emergence of HCC, our group performed longitudinal discriminant analysis of serial AFP measurements and developed a model to determine the risk of HCC using a large cohort of chronic liver disease undergoing surveillance using ultrasonography. Interestingly, average AFP levels tend to be elevated in those who had a history of HCC, more than 15 years before diagnosis. ${ }^{9}$ Therefore, the model can be used to assign patients to high and low-risk groups for late recurrence, and be further used to select patients for intensive surveillance after curative treatment.

In contrast, metabolic factors can also act in concert and contribute to the development of recurrent tumors. ${ }^{3,8}$ In this setting, recent knowledge regarding the mutational profiling of primary cancer and non-cancerous livers provides a clue to find the specific risk factors responsible for recurrence in each patient. ${ }^{10}$ Furthermore, a previous study has reported that viral exposure signature can predict the emergence of HCC among at-risk patients prior to a clinical diagnosis. Thus, exposure to both hepatitis virus and other viruses can affect hepatocarcinogenesis." For example, keratin strains of influenza virus trigger the emergence of $\mathrm{HCC}$, whereas human respiratory syncytial virus and human rhinovirus 23 are depleted in the HCC cohort. ${ }^{11}$ This might be useful in selecting patients with risk for late recurrence after curative treat-

Table 1. Phase 2 or 3 clinical trials of immune modulators in adjuvant setting after curative resection or radiofrequency ablation of HCC

\begin{tabular}{|c|c|c|c|c|}
\hline Setting & Trial identifier* & Agents (generic name) & Agents (type) & Number of participants $^{\dagger}$ \\
\hline \multicolumn{5}{|c|}{ Monotherapy } \\
\hline Phase 3 & NCT00149565 & Interferon alfa-2b & IFNa-2b & 268 \\
\hline Phase 3 & NCT03383458 (CheckMate 9DX) & Nivolumab & Anti-PD-1 antibody & 530 \\
\hline Phase 3 & NCT03867084 (KEYNOTE-937) & Pembrolizumab & Anti-PD-1 antibody & 950 \\
\hline Phase $2 / 3$ & NCT03859128 (JUPITER 04) & Toripalimab & Anti-PD-1 antibody & 402 \\
\hline Phase 2 & UMIN000026648 (NIVOLVE) & Nivolumab & Anti-PD-1 antibody & 55 \\
\hline \multicolumn{5}{|c|}{ Combinations } \\
\hline Phase 3 & NCT03847428 (EMERALD-2) & 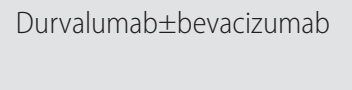 & $\begin{array}{l}\text { Anti-PD-L1 antibody } \pm \text { anti- } \\
\text { VEGFR2 antibody }\end{array}$ & 888 \\
\hline Phase 3 & NCT04102098 (IMbrave050) & Atezolizumab \pm bevacizumab & $\begin{array}{l}\text { Anti-PD-L1 antibody } \pm \text { anti- } \\
\text { VEGFR2 antibody }\end{array}$ & 662 \\
\hline Phase 2 & NCT03222076 & Nivolumab+ipilimumab & $\begin{array}{l}\text { Anti-PD-1 antibody+anti- } \\
\text { CTLA-4 antibody }\end{array}$ & 30 \\
\hline
\end{tabular}

HCC, hepatocellular carcinoma; IFN, interferon; PD-1, programmed cell death-1; PD-L1, programmed cell death-ligand 1; VEGFR2, vascular endothelial growth factor receptor 2 ; CTLA-4, cytotoxic T-lymphocyte antigen-4.

*The adjuvant IFNa-2b did not reduce the postoperative recurrence of viral hepatitis-related HCC (NCT00149565). Other trials including immune checkpoint inhibitors are currently ongoing.

${ }^{\dagger}$ Number of estimated enrollments.

‡Inclusion criteria show resectable hepatocellular carcinoma. 
ment of HCC.

For adjuvant therapy after curative treatment, several trials have been conducted to date and a few are still in process (Table 1). The result of phase 3 clinical trial that examines the efficacy of sorafenib in adjuvant setting revealed no difference in the recurrence-free survival between sorafenib and placebo groups. ${ }^{12}$ Other adjuvant agents, including interferon $\mathrm{a}-2 \mathrm{~b}$ and vitamin- $\mathrm{K} 2$, also failed to show promising results. ${ }^{13,14}$ Several reasons may contribute to the failure of developing adjuvant therapy in HCC cases. First, diversity in terms of molecular change may cause molecular heterogeneity in the recurrent tumors, ${ }^{15}$ which could induce refractoriness in specific agents even in the adjuvant setting. As aforementioned, patterns of molecular alterations completely differ among the metastatic recurrences of the primary lesion and de novo multicentric occurrences. Moreover, often these two recurrence types are hard to differentiate each other; these can co-exist in the same patients in the real clinical setting. Therefore, the heterogeneity of molecular alterations can be observed among different tumors in the same patients, ${ }^{15}$ which make it difficult to identify the suitable agents in adjuvant therapy, particularly for molecular targeted agents, because molecular heterogeneity induces tolerance for eliminating cancer cells. ${ }^{16}$ Second, notably, chronic inflammation, which is generally observed as a background condition of HCC, could induce immune tolerance in the liver. It is conceivable that induction of immune suppressive microenvironment will lead to high recurrence rate of HCC due to dysfunction of immune surveillance to cancer cells. ${ }^{17,18}$ Contentious induction of interferon- $\gamma$ could induce immune checkpoint molecules, and recruitment of regulatory $T$ cell, polarization of macrophages to immune suppressive M2 phenotype during healing process, and shifting the balance of helper T cell to immunosuppressive T-helper 2 phenotype occur during the progression of chronic inflammations. ${ }^{19,20}$ Collectively, these processes can contribute to the high recurrence rate of HCC after curative treatments compared to other cancers.

Because of the genetic heterogeneity of the recurrent tumor and background condition of immune tolerance that can result in the dysfunction of immune surveillance, immune modulators could be more suitable for adjuvant therapy compared to molecular targeted agents. ${ }^{20}$ From this point of view, currently, several phase 3 clinical trials for adjuvant therapy of HCC using immune checkpoint inhibitors are ongoing (Table 1).

Several risk factors can act in accordance and contribute to recurrence of HCC; these risk factors may be hard to eliminate because some may relate to the metabolic condition and others may be associated with environmental carcinogens and viral exposure, including non-hepatotropic virus. These conditions hamper the identification of subgroups related to recurrence, particularly for late recurrence, and development of adjuvant therapy after curative treatment. Nevertheless, recent comprehensive molecular and immunological knowledge may act as a powerful tool for developing effective surveillance system and adjuvant treatment in HCC after curative treatment.

\section{Conflicts of Interest}

The author has no conflicts of interests to disclose.

\section{REFERENCES}

1. Kanwal F, Singal AG. Surveillance for Hepatocellular carcinoma: current best practice and future direction. Gastroenterology 2019;157:54-64.

2. Shiina S, Tateishi R, Arano T, Uchino K, Enooku K, Nakagawa $H$, et al. Radiofrequency ablation for hepatocellular carcinoma: 10-year outcome and prognostic factors. Am J Gastroenterol 2012;107:569577; quiz 578.

3. Nahon P, Bourcier V, Layese R, Audureau E, Cagnot C, Marcellin P, et al. Eradication of hepatitis $\mathrm{C}$ virus infection in patients with cirrhosis reduces risk of liver and non-liver complications. Gastroenterology 2017;152:142-156.e2.

4. Kim J, Kang W, Sinn DH, Gwak GY, Paik YH, Choi MS, et al. Substantial risk of recurrence even after 5 recurrence-free years in early-stage hepatocellular carcinoma patients. Clin Mol Hepatol 2020;26:516-528.

5. Nishida N, Nishimura T, Kaido T, Minaga K, Yamao K, Kamata K, et al. Molecular scoring of hepatocellular carcinoma for predicting metastatic recurrence and requirements of systemic chemotherapy. Cancers (Basel) 2018;10:367.

6. Nishida N, Kudo M, Nagasaka T, Ikai I, Goel A. Characteristic patterns of altered DNA methylation predict emergence of human hepatocellular carcinoma. Hepatology 2012;56:994-1003.

7. Nishida N, Iwanishi M, Minami T, Chishina H, Arizumi T, Takita $M$, et al. Hepatic DNA methylation is affected by hepatocellular carcinoma risk in patients with and without hepatitis virus. Dig Dis 2015;33:745-750.

8. Nishida N, Yada N, Hagiwara S, Sakurai T, Kitano M, Kudo M. Unique features associated with hepatic oxidative DNA damage and DNA methylation in non-alcoholic fatty liver disease. J Gastroenterol Hepatol 2016;31:1646-1653.

9. Hughes DM, Berhane S, Emily de Groot CA, Toyoda H, Tada T, Kumada $T$, et al. Serum levels of a-fetoprotein increased more than 10 years before detection of hepatocellular carcinoma. Clin Gastroen- 
terol Hepatol. 2020 May 8. doi: 10.1016/j.cgh.2020.04.084.

10. Fujimoto A, Furuta M, Totoki $Y$, Tsunoda $T$, Kato $M$, Shiraishi $Y$, et al. Whole-genome mutational landscape and characterization of noncoding and structural mutations in liver cancer. Nat Genet 2016;48:500-509.

11. Liu J, Tang W, Budhu A, Forgues M, Hernandez MO, Candia J, et al. A viral exposure signature defines early onset of hepatocellular carcinoma. Cell 2020;182:317-328.e10.

12. Bruix J, Takayama T, Mazzaferro V, Chau GY, Yang J, Kudo M, et al. Adjuvant sorafenib for hepatocellular carcinoma after resection or ablation (STORM): a phase 3, randomised, double-blind, placebocontrolled trial. Lancet Oncol 2015;16:1344-1354.

13. Yoshida $H$, Shiratori $Y$, Kudo M, Shiina S, Mizuta T, Kojiro M, et al. Effect of vitamin $\mathrm{K} 2$ on the recurrence of hepatocellular carcinoma. Hepatology 2011;54:532-540.

14. Chen LT, Chen MF, Li LA, Lee PH, Jeng LB, Lin DY, et al. Long-term results of a randomized, observation-controlled, phase III trial of adjuvant interferon Alfa-2b in hepatocellular carcinoma after curative resection. Ann Surg 2012;255:8-17.
15. Kim SK, Takeda H, Takai A, Matsumoto T, Kakiuchi N, Yokoyama $A$, et al. Comprehensive analysis of genetic aberrations linked to tumorigenesis in regenerative nodules of liver cirrhosis. J Gastroenterol 2019;54:628-640.

16. Nishida N, Kitano M, Sakurai T, Kudo M. Molecular mechanism and prediction of sorafenib chemoresistance in human hepatocellular carcinoma. Dig Dis 2015;33:771-779.

17. Nishida N, Kudo M. Immune checkpoint blockade for the treatment of human hepatocellular carcinoma. Hepatol Res 2018;48:622-634.

18. Nishida N, Sakai K, Morita M, Aoki T, Takita M, Hagiwara S, et al. Association between genetic and immunological background of hepatocellular carcinoma and expression of programmed cell death-1. Liver Cancer 2020;9:426-439.

19. Nishida N, Kudo M. Immune phenotype and immune checkpoint inhibitors for the treatment of human hepatocellular carcinoma. Cancers (Basel) 2020;12:1274.

20. Brown ZJ, Greten TF, Heinrich B. Adjuvant treatment of hepatocellular carcinoma: prospect of immunotherapy. Hepatology 2019;70:14371442. 